\title{
Occurrence of adverse events in the activity of hospital wards in the opinions of doctors and nursing management staff
}

\author{
Marcin Mikos ${ }^{1, A-D}$, Aleksandra Banaszewska ${ }^{2, A-D}$, Halina Kutaj-Wąsikowska ${ }^{2, A-D}$, \\ Barbara Kutryba ${ }^{2, A-D}$, Aleksandra Czerw ${ }^{3, C, E-F}$, Anna Maria Badowska-Kozakiewicz ${ }^{4, E-F}$, \\ Ewa Wójtowicz ${ }^{2, E-F}$ \\ ${ }^{1}$ Department of Emergency Medical Services, Faculty of Medicine Andrzej Frycz Modrzewski Krakow University, Poland \\ ${ }^{2}$ National Center for Quality Assessment in Health Care, Krakow, Poland \\ ${ }^{3}$ Department of Health Economics and Medical Law, Medical University of Warsaw, Poland \\ ${ }^{4}$ Department of Biophysics and Human Physiology, Medical University of Warsaw, Poland
}

Mikos M, Banaszewska A, Kutaj-Wasikowska H, Kutryba B, Czerw A, Badowska-Kozakiewicz AM, Wójtowicz E. Occurrence of adverse events in the activity of hospital wards in the opinions of doctors and nursing management staff. Ann Agric Environ Med. $2020 ; 27(2)$ : $306-309$. doi: $10.26444 /$ aaem/106234

\begin{abstract}
Introduction. An adverse event is an incident induced while providing health care services or resulting from it, not related to the natural course of a given disease or health condition, which causes or is likely to cause negative consequences for the patient, including their death, a threat to life, the necessity of hospitalisation or its prolongation, permanent or considerable health detriment; or is a foetal disease, congenital defect or the result of foetal damage.

Objective. The aim of this analysis is to explore the problem of the occurrence of adverse events from the perspective of doctors and ward nurses who manage wards.

Materials and method. The research on the occurrence of adverse events among doctors and nurses (the management staff) was conducted with the use of a postal survey.

Results. It was ascertained that $86.5 \%$ of the medical personnel had taken part in an adverse event, of which $20.2 \%$ took part in an occurrence associated with pharmacotherapy, $16.2 \%$ - in an event related to diagnostics and diagnosis, or an infection $-15.7 \% .14 .2 \%$ of respondents were involved in an occurrence linked to a medical device malfunction, and $14.1 \%$ - in an adverse event related to an operation.

Conclusions. The adverse events most often identified in the nursing professional group are occurrences associated with pharmacotherapy, and in the doctors' professional group - occurrences related to diagnostics and diagnosis. The research established that the most frequent reason for not informing patients about the occurrence of an adverse event is fear of their filing a complaint. Medical management staff show high acceptance of an adverse event reporting system as a tool for improving patient safety.
\end{abstract}

\section{Key words}

adverse events, patient safety, quality of health care

\section{INTRODUCTION}

An adverse event is an incident induced while providing health care services or resulting from it, not related to the natural course of a given disease or health condition, which causes or is likely to cause negative consequences for the patient, including their death, a threat to life, the necessity of hospitalisation or its prolongation, permanent or considerable health detriment; or is a foetal disease, congenital defect or the result of foetal damage [1]. In Poland, adverse events affect around $7.2 \%$ of hospitalised patients [2]. The most frequent causes of adverse events in the treatment process, in the light of the analyses of court records of claims filed by patients, include the lack of medical personnel's due diligence in the performance of medical services $-21.38 \%$, a hospitalacquired infection in a patient $-15.86 \%$, medical personnel errors $-9.66 \%$, as well as irregularities in treatment $-3.45 \%$

Address for correspondence: Marcin Mikos, Department of Emergency Medical Services, Faculty of Medicine, Andrzej Frycz Modrzewski Krakow University, Poland, Poland

E-mail:mikos@ziz.com.pl

Received: 26.02.2019; accepted: 09.04.2019; first published: 15.04.2019
[3]. Among the most common adverse events, the following are indicated: a foreign body left in an operative field, wrong patient/area/side operated on/wrong operating procedure, a catheter-related infection of the vascular bed, bodily injuries resulting from an operation, sepsis after surgery, pulmonary embolism or deep vein thrombosis after surgery, suicide in hospital, improper administration of medication (wrong medicine/dose/patient/administration time/route), falls in hospital, failure to deliver timely care, revision surgeries, unplanned, recurrent hospitalisations or leaving hospital arbitrarily (against medical advice) by a patient [4].

Adverse event reporting systems are the basic tool for improving patient safety. Thanks to their functioning, medical personnel have the ability to identify adverse events which, after being revealed and properly analysed, are a basis for searching for their causes, and subsequently for both taking corrective actions in particular entities and reducing the systemic causes. In order for a reporting system to be a tool for the effective improvement of patient safety, it should comply with the minimal conditions determined by experts and should: 
- by definition, not be punitive or unjust;

- be independent of other legislative processes;

- create conditions for health care providers and medical personnel which stimulate them to notify adverse events (i.e. voluntariness, anonymity, confidentiality - wherever possible);

- constitute a system of collecting and reporting data concerning adverse events at local level and, if necessary, aggregating data to improve patient safety at regional or national level;

- encompass both the private and public sectors;

- facilitate the engagement of patients, their relatives and informal caregivers in all aspects of patient safety actions, including the notification of adverse events [4].

The basic condition for the effective reporting of adverse events by medical personnel is the absence of sanctions for the involvement in an incident and for reporting it. Under the current state of law, this condition is not fulfilled in the health care system in Poland.

Currently, in Poland, adverse event reporting systems are not a compulsory solution, and are only a voluntary practice applied by a part of medical facilities. Obligatorily, the reporting of adverse events is conducted by the hospitals accredited by the Ministry of Health with respect to quality. The standard regarding the quality and patient safety improvement (PJ5) assumes, among other things, identifying adverse events and collecting data on them in an accredited hospital, analysing adverse events related to a patient's hospital stay, and taking advantage of the conclusions from the conducted analyses of adverse events in hospital management [5].

The study shows that accreditation of hospitals constitutes an effective way of verifying and shaping the quality and safety of health care services provided in hospitals, through organisational results, such as, among others, the standardisation of personnel activities and conduct, creating a quality monitoring system by setting measurable indicators, the regulation and reorganisation of hospital activity with regard to respecting patients' rights, the improvement of human resources management, regulation of competencies and responsibilities, and a patient-oriented approach, with the patient being the main subject in health care activities [6].

\section{OBJECTIVE}

The aim of this study is to explore the problem of the occurrence of adverse events from the perspective of doctors who manage wards, and from the perspective of ward nurses.

\section{MATERIALS AND METHOD}

The research, aimed at exploring the problem of the occurrence of adverse events from the perspective of doctors who manage wards and from the perspective of ward nurses, was conducted in surgical and non-surgical units. The survey covered 1,705 departments in 557 hospitals of different referral levels, accredited by the Ministry of Health, or lacking such an accreditation. A total of 3,410 questionnaires were sent to respondents, of which 1,864 completed questionnaires $(54.7 \%)$ were returned. 990 questionnaires came from

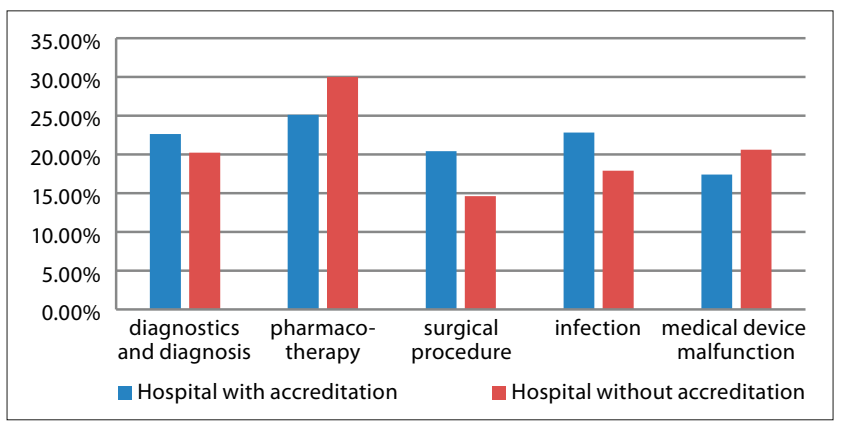

Figure 1. Adverse events identified in hospitals accredited by the Ministry of Health, compared with non-accredited hospitalks [7]

surgical wards (53.1\% of the total) and 817 from non-surgical wards $(43.8 \%)$. Nearly $60 \%$ of the questionnaires $(57.8 \%)$ were filled-in by employees in hospitals with a certificate of accreditation from the Ministry of Health, and over one-third of them (35.6\%) in non-accredited hospitals. In other cases, respondents had no knowledge of whether a given facility had accreditation from the health minister (Fig. 1) [7].

Data were presented as the number of cases and percentages (\%). Chi-squared test was employed to evaluate differences in response rates and a p-value of 0.05 was considered statistically significant. All calculations were conducted using STATISTICA version 12.0 (StatSoft, Inc., Tulsa, OK, USA).

\section{RESULTS}

Types of adverse events. Adverse events in which the medical personnel taking part in the survey were most frequently involved, included incidents associated with pharmacotherapy (20.2\%), diagnostics and diagnosis (16.2\%), an infection (15.7\%) and a medical device malfunction (14.2\%). $14.1 \%$ of the survey participants were exposed to a surgery-related adverse event.

The study ascertained that medical management staff of facilities with an accreditation certificate from the Ministry of Health more often indicated that they were faced with adverse events related to operations $(15.7 \%$, while in the non-accredited ones - $10.7 \%)$ and infections (17.5\%, while in the non-accredited - 13.1\%); the difference was statistically significant (respectively: $\mathrm{p}=0.002 ; \mathrm{p}=0.009$ ).

Among nurses, an identified group of adverse events in which the representatives of this profession most often took part comprised occurrences associated with pharmacotherapy (22.1\%), an infection (14.3\%) and medical device malfunctions (11.0\%).

Among doctors, an identified group of adverse events in which the representatives of that profession most commonly took part consisted of events related to diagnostics and diagnosis (25.3\%), operations (23.3\%), and adverse events linked to pharmacotherapy (18.6\%).

Causes of adverse events. The most frequent cause of hospital avoidable adverse events is the excessive workload of the medical personnel. This view was expressed by $87.5 \%$ of those taking part in the survey. Among the indicated potential causes of avoidable adverse events, the following were pointed out, amongs: lack of sufficient motivation in the professional group of nurses, lack of sufficient education and training, lack of the habit of mutual criticism, and lack of sufficient 
support for more experienced colleagues. Employees of non-accredited hospitals more often pointed out outdated or incomplete procedures - $30.4 \%$ (18.4\% in accredited hospitals) and lack of sufficient education and training $37 \%$ (26.4\% in accredited hospitals). The differences were significant (respectively: $\mathrm{p}<0.001$ and $\mathrm{p}<0.001$ ).

Attitudes of medical personnel to adverse events. $79.3 \%$ of the survey participants confirmed that the personnel of their wards are open to the notification of adverse events. A similar result was obtained regarding the conviction that in a given hospital unit, assistance for the personnel involved in an adverse event is ensured $-74.2 \%$. Opinions regarding the attitudes of medical personnel in a ward to reporting adverse events did not vary significantly between hospitals with a certificate of accreditation and those without the certificate.

The study showed that the most frequent causes for not informing patients about the occurrence of an adverse event by medical personnel are as follows: the fear of a patient filing a complaint $-25.2 \%$ of the respondents surveyed; fear of unpleasant contact with a patient's family - $15.1 \%$, and also the potential possibility of media intervention $-11.9 \%$.

Adverse event reporting systems. The vast majority - $85 \%$ of respondents - agreed with the statement that the introduction of an adverse event reporting system would improve patient safety. The conditionally confidential model is the adverse event reporting model that gained the highest acceptance $45.7 \%$ of the medical personnel taking part in the survey. The model of the anonymous reporting of adverse events has the lowest acceptance of the medical personnel participating in the survey. In the majority of cases (68.2\% of respondents), the survey participants opted for the mandatory nature of adverse event reporting, based on a list of adverse events subject to obligatory reporting.

\section{DISCUSSION}

Providing medical services by doctors and nurses is accompanied by adverse events, which are a significant medical, organisational and economic burden for health care systems, and also for social welfare systems. In some countries, treatment costs alone of the aftereffects of damage resulting from adverse events reach $9.5 \%$ of total health expenditure [8].

In the majority of studies, hospital infections constitute the main group of identified adverse events. In the UK, for instance, around 1 in 20 hospitalised patients experience such a complication. The most common are urinary tract $(27 \%)$, lower respiratory tract (24\%), perioperative (17\%) and bloodborne infections (10.5\%) [9]. This trend does not correspond with the result obtained in the presented study in which hospital infections were indicated as only the third group of the most numerous adverse events, after incidents related to pharmacotherapy and those associated with diagnostics and diagnosis.

Adverse event reporting systems are currently the most effective tool for detecting and preventing adverse events. However, the notification of incidents must take place with the support of medical personnel, who need to be convinced of the advisability and effectiveness of using such a tool.

The study ascertained that adverse event reporting has high support among medical management staff and, in their view, is a tool for the effective identification and prevention of adverse events, in particular in facilities having quality accreditation from the Ministry of Health, which proves that this type of certification serves the quality and patient safety improvement, compared to hospitals without accreditation [6].

Results regarding the attitudes of medical personnel towards patients in a situation where an adverse event occurs obtained in the research, and the difficulties of informing a patient about an incident in particular, remain identical with opinions of patients. In a public opinion poll in this field, $27 \%$ of the interviewees received information about the occurrence of an adverse event from medical personnel, and causes of events were explained by medical personnel to $13 \%$ of patients. An apology was offered to a patient in $12 \%$ of situations, and in $9 \%$ of situations, a patient was informed about potential consequences of an adverse event [10]. It was indicated in the literature on this subject that paternalistic attitudes of the medical personnel negatively affect relations with recipients of the services, including the decrease of patient engagement in shared decision making with regard to a health care procedure which, consequently, has a subsequent negative influence on patient safety [11].

In the current study, the medical personnel surveyed indicated fatigue as being the major cause of the occurrence of adverse events among medical personnel in hospitals. The result obtained is identical to numerous reports in the scientific literature that confirms the existence of this correlation [12].

\section{CONCLUSIONS}

In hospitals which have an accreditation in health care from the Ministry of Health, senior medical staff identify around $5 \%$ more adverse events related to operations and hospital infections than in hospitals without this accreditation.

1. In the nursing professional group, the most frequently identified advert events include occurrences associated with pharmacotherapy, and in the professional group of doctors - events linked to diagnostics and diagnosis.

2 . The dominant cause of the occurrence of advert events in hospital wards, regardless of professional group, is fatigue among the medical personnel.

3. Among doctors and nurses fulfilling managerial functions, there is high support for the introduction of an adverse event reporting system as a tool which effectively improves patient safety. The majority of the survey participants were of the opinion that notification of advert events should, however, be mandatory and conducted according to the conditionally confidential model.

4. In spite of the openness of doctors and nurses who fulfill managerial functions to reporting adverse events, there are significant barriers to informing patients about the occurrence of an adverse event for fear of the demanding attitude of the patients.

\section{REFERENCES}

1. Guidelines for the quality in health care and patient safety bill), source: www.legislacja.gov.pl accessed: 21.11.2017.

2. National Center for Quality Assessment in Health Care, source: ww.cmj. org.pl accessed: 22.11.2017. 
3. The research was conducted within the framework of the 'Safe Hospital - Safe Patient' project co-ordinated by the National Center for Quality Assessment in Health Care.

4. Explanatory memorandum of the Council of Europe, Committee of Experts on Management of Safety and Quality in Health Care Prevention of adverse events in health care, a system approach (SP-SQS), Strasburg, 1 April 2005.

5. A list of quality standards for hospitals) - source: National Center for Quality Assessment in Health Care, www.cmj.org.pl downloaded: 23.11.2017.

6. Szczurek T. Using accreditation to ensure the quality and safety of medical services, Science Notebooks, UEK, 2015; 1(948): 65-80.

7. A survey into the opinions of doctors and nursing personnel about adverse event reporting and requirements which should be fulfilled by reporting systems in health care), Polish Society for Quality Promotion in Health Care, commissioned by the National Center for Quality Assessment in Health Care, Kraków 2015.

8. Lim SJ, Chung WJ, Cho WH. Economic burden of injuries in South Korea, Inj Prev. 2011 Oct; 17(5): 291-6. doi: 10.1136/ip.2010.028118. Epub 2011 May 27.

9. Department of Health. An organisation with a memory. Report of an expert group on learning from adverse events in the NHS. London, Publisher: Crown, 2000.

10. Research of the Public Opinion Research Centre (CBOS) commissioned by the National Center for Quality Assessment in Health Care - Adverse events and the perception of health care safety, 2015.

11. Longtin Y. Patient participation: current knowledge and applicability to health care safety). Med Dypl. 2010; 19: 6 .

12. Jennings BM, Sandelowski M, Mark B. The nurse's medication day. Qual Health Res. 2011; 21: 1441-51. 